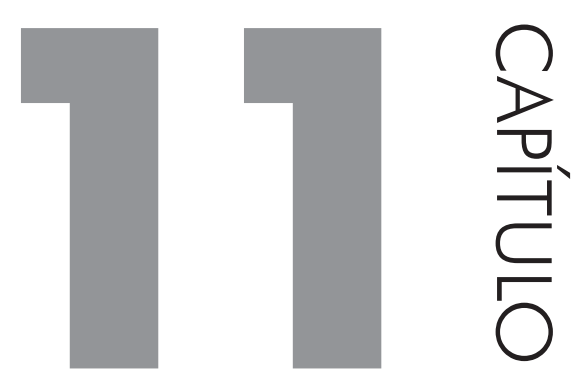

\title{
MOODLE COMO FERRAMENTA DE SUPORTE A PBL EM REDE: UMA REVISÃO SISTEMÁTICA
}

\section{GIOVANNI FERREIRA DE FARIAS, NILDA MARIA DE MEDEIROS BRITO FARIAS, FERNANDO JOSÉ SPANHOL, MÁRCIO VIEIRA DE SOUZA}

\section{INTRODUÇÃO}

Aprendizado Baseado em Problemas, metodologia mais conhecida pela sua denominação em inglês Problem Based Learning (PBL), tem sua origem oriunda da Universidade McMaster, Canadá, no final dos anos 60 (Albanise \& Michael, 1993). Trata-se de uma metodologia centrada no aluno, usada em praticamente todos os níveis educacionais, em que o ensinamento é baseado na solução de problemas efetivamente ou supostamente reais em que são empregados os conceitos a serem aprendidos pelos alunos, fazendo com que os mesmos desenvolvam as competências necessárias ao aprendizado (Leon \& Onófrio, 2015). O processo de aprendizado é realizado em pequenos grupos de alunos, que realizam as atividades necessárias para solução de um problema dado pelo professor. Isso implica em, numa primeira sessão, analisarem o problema para obter seu entendimento, identificarem e buscarem os conhecimentos e competências necessárias para a solução do mesmo. Partem então para um trabalho externo à sala de aula, com papéis bem definidos de trabalho individual e/ou em equipe. Em uma segunda sessão, reúnem-se novamente com o professor para propor e/ou executar a solução encontrada, discutindo com a propriedade da solução e ocasionalmente tendo complementação do tutor. 


\section{EMBASAMENTO TEÓRICO}

Segundo Wood (2003), Borrows (1996), Leon \& Onófrio (2015) e Albanise \& Michael (1993), a metodologia PBL visa desenvolver no aluno algumas importantes competências relacionadas com o aprendizado, tais como: desenvolver uma larga base de conhecimento, adquirir competências para efetiva solução de problemas, se motivar ao aprendizado, aprender a aprender autonomamente, bem como a trabalhar eficientemente em equipe. Enfim, o aluno acaba desenvolvendo o que Woods (2006) chama de competência em conceitos, relacionados com a teoria que deve aprender, bem como o que chama de competência em processos, referente à forma como se aprende os conceitos, tais como: liderança, resolução de conflitos, colaboração, comunicação, entre outros.

Já estabelecida como metodologia de aprendizagem de resultados efetivos, PBL vem sendo usada em programas inteiros de cursos de graduação ao redor do mundo, especialmente na área de Saúde. A metodologia passou a contar com ajuda da tecnologia da informação e comunicação para implementar seus processos, especialmente a partir do final dos anos 90 (Farias, Spanhol \& Souza, 2017; Ali, Samaka \& Shaban, 2011). Inicialmente houve uma proliferação de softwares desenvolvidos especificamente para implementação de aprendizagem baseada em problemas, não necessariamente seguindo a fundamentação da metodologia preconizada por Woods (1994) ou Berkel et al. (2010). Pois o uso de alguns destes softwares sequer implicava em atividades desenvolvidas em equipe ou com tutoria, a aprendizagem se baseava em problemas elencados a partir de trilhas de questões, com respostas predefinidas e correção automatizada (Miao, Holst, Holmer, Fleschutz \& Zentel, 2000; Subnukarn \& Haddawy, 2007; Chan, 2006).

Com a popularização dos ambientes virtuais de aprendizagem, surgiram várias iniciativas do uso deste tipo de ferramenta para implementação de PBL em rede, ou seja, através de uso de ferramentas baseadas na Internet para implementar pelo menos parte dos processos PBL (Ali, Samaka \& Shaban, 2011). Segundo Farias, Spanhol \& Souza (2016), a literatura apresenta quatro grandes enfoques quando se trata de ambientes virtuais de aprendizagem e a metodologia PBL: modelos para desenho de atividades PBL através da Internet, avaliação de tecnologias que dão suporte à implementação da metodologia, avaliação dos resultados de experimentos com uso de PBL em rede, e uso de plug-ins para otimizar ambientes virtuais de aprendizagem no contexto de PBL em rede. Apesar destacar o Moodle (Modular Object Oriented Dynamic Learning Environment) dentre os ambientes virtuais de aprendizagem utilizados no suporte à PBL em rede, estando presente na maioria das publicações levantadas no estudo, o estudo não deu um maior enfoque sobre como o Moodle é utilizado nestas implementações. 
Desta forma, este estudo tem como objetivo primário analisar a literatura para entender como especificamente o Moodle é utilizado no contexto de implementação da metodologia Problem Based Learning em rede. Como objetivos secundários, este estudo visa identificar a abrangência de uso do Moodle para a implementar PBL, ou seja, para o desenvolvimento dos alunos em competências em conceitos e em processos citados por Woods (2006); além de verificar a adequação e/ou limitações do Moodle na implementação de PBL em rede de acordo com os relatos encontrados.

\section{METODOLOGIA DE TRABALHO}

Este estudo propõe a execução de uma revisão sistemática sobre o tema proposto, com análise qualitativa das publicações, baseada numa epistemologia interpretativista (Crotty, 2010, p. 98). Os critérios de seleção das publicações são apresentados abaixo:

- Argumento de busca booleana: (“PBL” ou "ABP” ou "Problem Based Learning" ou "Aprendizagem Baseada em Problemas" ou "Aprendizaje Basada em Problemas") e Moodle.

- Bases de dados utilizadas: Scopus, Web of Science, EBSCO e Scielo.

- Campos pesquisados: título, resumo e palavras-chave.

- Idiomas: português, inglês e espanhol.

- Tipos de publicações: revistas indexadas e anais de congressos.

- Período: publicações realizadas até 2016 (inclusive).

Após a leitura na íntegra de todas as publicações selecionadas, foram excluídas aquelas que passaram indevidamente pela filtragem dos engenhos de busca das bases de dados supracitadas, bem como as publicações cujo teor era não apresentavam a maioria dos principais aspectos da metodologia Problem Based Learning: apresentação de problemas mal estruturados para posterior análise e planejamento/discussão em grupo sobre solução por parte dos alunos, com acompanhamento de tutoria e avaliação pelos pares ou autoavaliação. (Albanise \& Michael, 1993; Woods, 2006)

Foram particularmente identificadas e excluídas desta pesquisa as publicações cujo enfoque principal era a metodologia Project Based Learning (aqui citado como PjBL para diferenciar da sigla PBL), que apesar da semelhança no nome, na realidade do contexto de estudo e de alguns procedimentos de implementação, difere de PBL nos encaminhamentos e objetivos.

Quando se trata de PjBL, o foco do aprendizado é se chegar a um produto ou artefato, como um material de apresentação ou um software, que pode ser desenvolvido em grupo ou individualmente, seguindo um passo-a-passo genérico 
e variável. Já quando se trata de PBL, o foco do aprendizado é se alcançar uma solução do problema e não a criação de um artefato para tal solução, sendo obrigatoriamente realizado em grupo, e seguindo um passo-a-passo mais rígido e clássico. Além do mais, ao contrário do que ocorre com PjBL, no PBL é dado um maior enfoque ao processo de aprendizagem, ou seja, sobre como se aprende, especialmente no que diz respeito à competências de trabalho em grupo. (Farias, Spanhol \& Souza, 2017, p. 43)

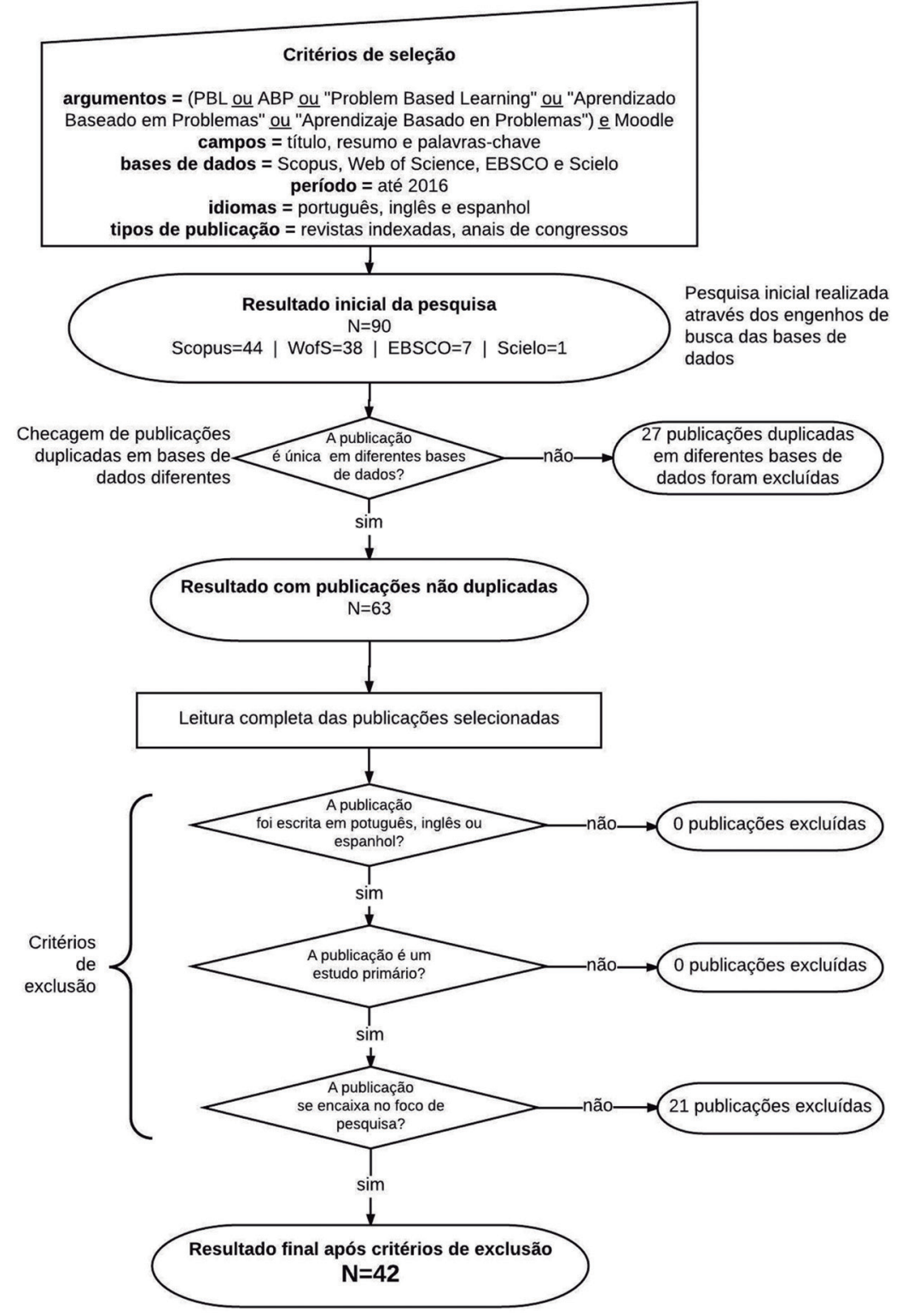

Figura 1 Esquema da pesquisa sistemática realizada neste estudo. 


\section{RESULTADOS E BIBLIOMETRIA}

A Figura 1 ilustra os processos de busca, seleção e exclusão realizados nesta pesquisa. Como resultado da revisão sistemática, foram levantadas 63 publicações para análise, das quais 43 se mostraram adequadas aos critérios estabelecidos para este estudo. Os motivos que ocasionaram as 20 exclusões foram diversos: algumas publicações utilizavam algum dos argumentos de busca mas não tinham qualquer relação com o tema desta pesquisa (5), outras faziam alusão ao Moodle em PBL apenas para compará-lo com uma tecnologia proprietária (2), outras 2 publicações partiam do entendimento de PBL se baseava meramente na solução de problemas (ex.: questões de matemática) com avaliação somativa feita com questionários do Moodle, enquanto a maioria delas (11) efetivamente tratavam de PjBL, embora algumas delas declarassem tratar de PBL.

Para facilitar a visão geral sobre as publicações que compuseram este estudo, a Tabela 1 apresenta a lista das referências junto com informações consideradas importantes para o trabalho de análise, com sinalização através de ícones para facilitar a leitura, como listado abaixo.

- Citação da referência.

- Área do conhecimento envolvida no estudo, de acordo com a classificação realizada pela Capes (2017), exceto quando se tratava de educação básica ou de descrição de um produto.

- País de origem da publicação.

- Elementos-chave identificados no texto da publicação, indicados por ícones letras: avaliação por pares ou auto avaliação (Ap), seja de conceitos ou de processos; uso do Moodle para distribuição de conteúdo $(\mathrm{Ct})$, realização de interatividade entre alunos e tutores (In), especialmente quando mediada por Wikis (W em negrito), ou realização de avaliação (Av); texto alusivo a $\mathrm{PjBL}$ embora o procedimento fosse típico de $\mathrm{PBL}(\mathrm{Pj})$; indicação explícita de resultados (positivos) sobre a implementação de PBL em rede (Rp), com eventual indicativo de alguma ressalva $\left(\mathrm{Rp}^{*}\right)$. Não houve resultados apresentados como negativos no uso de PBL em rede com o Moodle.

Tabela 1 Listagem das referências bibliográficas levantadas na pesquisa sistemática.

\begin{tabular}{|l|l|l|l|l|l|l|l|l|}
\hline \multicolumn{1}{|c|}{ REFERÊNCIA } & \multicolumn{1}{c|}{$\begin{array}{c}\text { ÁREA DE } \\
\text { CONHECIMENTO }\end{array}$} & \multicolumn{3}{c|}{ PAís } & \multicolumn{3}{c|}{ ELEMENTOS CHAVE } \\
\hline Glacomozzi et al. 2006 & Ciências biológicas & Itália & & Ct & In & & & \\
\hline Glacomozzi et al. 2008 & Ciências biológicas & Itália & & Ct & In & & & \\
\hline Huang e Chuang, 2008 & Educação básica & Taiwan & & & In & & & Rp \\
\hline $\begin{array}{l}\text { Boldini, Bracchini, Pouyouta, Solomou e e } \\
\text { loannou, 2009 }\end{array}$ & Educação básica & Itália & Ap & Ct & In & Av & & \\
\hline
\end{tabular}




\begin{tabular}{|c|c|c|c|c|c|c|c|c|}
\hline $\begin{array}{l}\text { Garcia-Robles, Diaz-del-Rio, Vicente-Diaz e } \\
\text { Linhares-Barranco, } 2009\end{array}$ & Ciências exatas & Espanha & Ap & $\mathrm{Ct}$ & & & & $\mathrm{Rp}$ \\
\hline Saydam, Mitra, Daly e Hagan, 2009 & Engenharia & Austrália & Ap & Ct & $\ln$ & $A v$ & $\mathrm{Pj}$ & \\
\hline Barnard-Ashton, 2010 & Ciências biológicas & África do Sul & & $\mathrm{Ct}$ & $\mathbf{w}$ & & & $\mathrm{Rp}^{*}$ \\
\hline Diez-Higuera et al. 2010 & Engenharia & Espanha & Ap & & & Av & $\mathrm{Pj}$ & $\mathrm{Rp}$ \\
\hline $\begin{array}{l}\text { Gomez-Lahoz, Garcia-Rubio, Paz Garcia, } \\
\text { Rodriguez-Maroto, Garcia-Herruzo e Vereda- } \\
\text {-Alonso, } 2010\end{array}$ & Ciências exatas & Espanha & Ap & $\mathrm{Ct}$ & In & $A v$ & $\mathrm{Pj}$ & Rp \\
\hline Halbaut et al, 2010 & Ciências biológicas & Espanha & Ap & $\mathrm{Ct}$ & & Av & & $\mathrm{Rp}$ \\
\hline Mezzari, 2010 & Ciências biológicas & Brasil & & $\mathrm{Ct}$ & $\ln$ & $A v$ & & \\
\hline Podesta, 2010 & Engenharia & Itália & & $\mathrm{Ct}$ & In & $A v$ & & $\mathrm{Rp}^{*}$ \\
\hline Ali, Samaka e Shaban, 2011 & Descrição de produto & Catar & Ap & $\mathrm{Ct}$ & $\ln$ & Av & $\mathrm{Pj}$ & \\
\hline $\begin{array}{l}\text { Alonso, Arriba, Iniesta, Herrera, Santa Cruz } \\
\text { e Sanz, } 2011\end{array}$ & Ciências biológicas & Espanha & & $\mathrm{Ct}$ & In & & & \\
\hline Martinez, Herrera e Pablo, 2011 & Engenharia & Espanha & & $\mathrm{Ct}$ & $\mathbf{w}$ & Av & $\mathrm{Pj}$ & $\mathrm{Rp}$ \\
\hline $\begin{array}{l}\text { Moreno-Brea, Arellano-Lopez e Arufe- } \\
\text {-Martinez, } 2011\end{array}$ & Ciências biológicas & Espanha & Ap & & $\mathbf{w}$ & Av & & Rp \\
\hline Osinaga Mujika e Manso, 2011a & Ciências exatas & Espanha & Ap & $\mathrm{Ct}$ & $\mathbf{w}$ & & $\mathrm{Pj}$ & $\mathrm{Rp}$ \\
\hline Osinaga Mujika e Manso, 2011b & Ciências exatas & Espanha & Ap & $\mathrm{Ct}$ & $\mathbf{w}$ & & $\mathrm{Pj}$ & $\mathrm{Rp}$ \\
\hline $\begin{array}{l}\text { Regueiro-Gomez, Busoch-Morlin, Busoch e } \\
\text { Sanchez-Bao, } 2011\end{array}$ & Engenharia & Cuba & & $\mathrm{Ct}$ & $\mathbf{w}$ & & $\mathrm{Pj}$ & \\
\hline $\begin{array}{l}\text { Sancho, Torrente, Marchiori e Fernandez- } \\
\text {-Manjn, } 2011\end{array}$ & Descrição de produto & Espanha & Ap & $\mathrm{Ct}$ & In & & & \\
\hline Zheng e Zhou, 2011 & Ciências exatas & Hong-Kong & & & $\ln$ & Av & & $\mathrm{Rp}^{*}$ \\
\hline Audi e Escudero, 2012 & Ciências sociais aplicadas & Espanha & & $\mathrm{Ct}$ & $\ln$ & Av & $\mathrm{Pj}$ & $\mathrm{Rp}$ \\
\hline Gutierrez e Berciano, 2012 & Ciências exatas & Espanha & Ap & & & Av & & Rp \\
\hline Sancho, Torrente e Fernandez, 2012 & Engenharia & Espanha & & & $\mathbf{w}$ & & & $\mathrm{Rp}$ \\
\hline Sevil, Terres e Llanas, 2012 & Engenharia & Espanha & & $\mathrm{Ct}$ & & & $\mathrm{Pj}$ & Rp \\
\hline Shober e Keller, 2012 & Educação básica & Áustria & Ap & $\mathrm{Ct}$ & & & & $\mathrm{Rp}$ \\
\hline $\begin{array}{l}\text { Stebbings, Bagheri, Perrie, Blyth \& McDonald, } \\
2012\end{array}$ & Ciências biológicas & Nova Zelândia & Ap & Ct & In & Av & & $\mathrm{Rp}$ \\
\hline Ali e Samaka, 2013 & Descrição de produto & Catar & Ap & $\mathrm{Ct}$ & $\ln$ & Av & $\mathrm{Pj}$ & \\
\hline $\begin{array}{l}\text { Gonzalez-Sancho, Sanchez-Pacheco, Lasa, } \\
\text { Molina, Vara e Del Peso, } 2013\end{array}$ & Ciências biológicas & Espanha & Ap & Ct & In & $A v$ & & Rp \\
\hline Tiantong e Teemuangsai, 2013 & Ciências exatas & Tailândia & & $\mathrm{Ct}$ & $\ln$ & & $\mathrm{Pj}$ & \\
\hline Coquerel et al. 2014 & Ciências biológicas & Brasil & & & & Av & & $\mathrm{Rp}$ \\
\hline Onan, Turan e Gurlen, 2014 & Ciências exatas & Turquia & & $\mathrm{Ct}$ & $\mathbf{w}$ & & & $\mathrm{Rp}$ \\
\hline $\begin{array}{l}\text { Reis, Ikari, Taha-Neto, Gugliotta e Denardi, } \\
2014\end{array}$ & Ciências biológicas & Brasil & & $\mathrm{Ct}$ & & & & \\
\hline
\end{tabular}




\begin{tabular}{|l|l|l|l|l|l|l|l|l|}
\hline Tirado \& Santos, 2014 & Ciências humanas & México & Ap & Ct & $\mathbf{W}$ & & & Rp \\
\hline Ai-Dous e Samaka, 2015 & Descrição de produto & Catar & Ap & Ct & In & Av & Pj & \\
\hline Ali, Al-Dous e Samaka, 2015 & Descrição de produto & Catar & Ap & Ct & In & Av & Pj & \\
\hline Bhardwaj, Nagandla, Swe e Abas, 2015 & Ciências biológicas & Malásia & & Ct & In & Av & & \\
\hline Biasutti e El-Deghaldy, 2015 & Ciências humanas & Itália & Ap & Ct & $\mathbf{W}$ & $\mathrm{Av}$ & $\mathrm{Pj}$ & $\mathrm{Rp}$ \\
\hline Fernandez-Borrs, Torrela, Viscor e Pages, 2015 & Ciências biológicas & Espanha & & & & $\mathrm{Av}$ & & $\mathrm{Rp}$ \\
\hline $\begin{array}{l}\text { Mart, Gil, Gurgu, Hernandez-Sabat, Rocaras } \\
\text { e Poveda, 2015 }\end{array}$ & Engenharia & Espanha & $\mathrm{Ap}$ & $\mathrm{Ct}$ & $\mathrm{In}$ & $\mathrm{Av}$ & $\mathrm{Pj}$ & $\mathrm{Rp}$ \\
\hline Sein-Echaluce et al. 2015 & Ciências exatas & Espanha & $\mathrm{Ap}$ & $\mathrm{Ct}$ & $\mathbf{W}$ & $\mathrm{Av}$ & $\mathrm{Rp}$ \\
\hline Ali, Wang, Samaka e Miao, 2016 & Descrição de produto & Catar & $\mathrm{Ap}$ & $\mathrm{Ct}$ & $\mathrm{In}$ & $\mathrm{Av}$ & $\mathrm{Pj}$ & \\
\hline
\end{tabular}

\section{ANÁLISE E DISCUSSÃO DOS DADOS}

Como pode ser visto, o primeiro achado da pesquisa, ocorrido ainda na fase de exclusão de referências para compor o acervo de estudo, foi sobre a falta de uma clareza do que implica proceduralmente a metodologia PBL por parte dos autores. Isso foi notório especialmente quando percebida a confusão entre PBL e PjBL. Esta confusão não se refletiu apenas nas publicações excluídas do estudo, mas também em algumas das publicações que foram incluídas no mesmo.

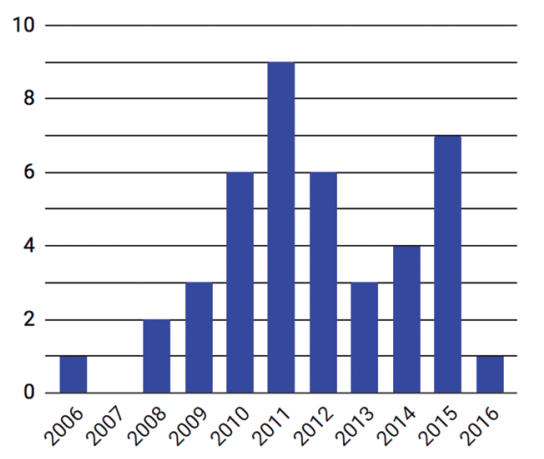

(a) Número de publicações selecionada por cada ano.

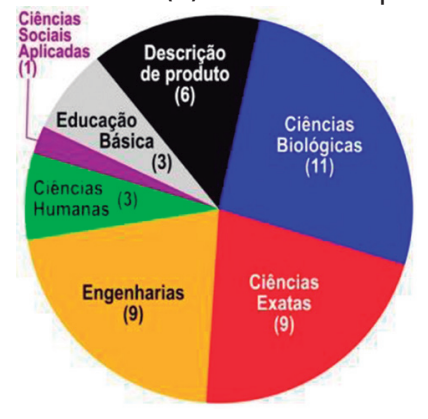

(b) Publicações por área do conhecimento.

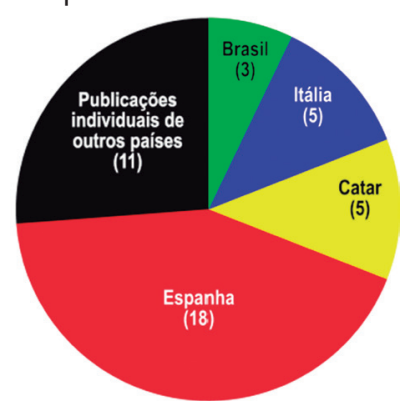

(c) Produção de países com pelo menos duas publicações.

Figura 2 Gráficos bibliométicos da pesquisa sistemática. 
Há publicações em envolvem a elaboração de projetos pelos alunos ou envolvimento de artefatos a serem tratados no processo de aprendizagem, o que a princípio leva a crer que se trata de PjBL. Outras publicações chegam a explicitar no título do trabalho, que fazem uso de Project Based Learning. Porém, uma análise cuidadosa do texto deixa claro que fizeram uso de Problem Based Learning, e por incluírem projetos nas atividades foram denominadas PjBL. Para estes casos, a respectiva publicação foi marcada com o ícone correspondente $(\mathrm{Pj})$ na Tabela 1.

As publicações incluídas na pesquisa com alguma menção à $\mathrm{PjBL}$ mas que eram PBL, bem como as publicações que foram excluídas por se definirem como relacionadas com PBL mas sendo, de fato, PjBL, demonstram a falta de consenso entre os autores na distinção entre aprendizagem baseada em problemas e em projetos. Alguns casos demonstram muito claramente essa falta de padronização sobre o que se define por PBL. Diez-Higuera et al. (2010, p. 1307), por exemplo, define Problem Based Learning como sendo uma variante de Project Based Learning, sem qualquer referencial que dê suporte a tal definção. Já Stebbings (2012, p. 1180) descreve "seminários PBL" como sendo conteúdo rico para ser acessado pelos alunos antes de iniciar as atividades PBL. Em ambos os casos, o entendimento dos autores sobre PBL não encontra respaldo na descrição da metodologia dentre publicações de referência na área, como Woods (2006), Barrows (1996), Albanise \& Michael (1993) ou Berkel et al. (2010).

Outra indicação que falta alinhamento sobre os procedimentos a serem seguidos na implementação de PBL é a ausência de avaliação por pares ou auto-avaliação, seja sobre conceitos ou sobre processos. Como pode ser visto na Tabela 1, o ícone referente a este procedimento (Ap), surge apenas 23 publicações, ainda assim devido ao esforço feito na leitura dos textos para identificar que havia algum tipo de avaliação por pares ou autoavaliação nos procedimentos descritos, pois nem sempre isso era explicitamente citado. Na maior parte dos textos, o que efetivamente é explicitado é apenas a apresentação de problemas para discussão entre os alunos, para posterior formulação de uma solução, mas nem sempre fica clara a avaliação de conceitos ou de processos, feita por pares ou pelo próprio aluno.

Outros três ícones utilizados na Tabela 1 são diretamente relacionados com os recursos do Moodle, indicados em preto na tabela quando a publicação indica o uso de recursos para publicação de conteúdo $(\mathrm{Ct})$, para interatividade entre os participantes (In|W) e para avaliação do aprendizado (Av). A análise do uso do Moodle com essa classificação foi importante para saber a abrangência do uso do ambiente virtual de aprendizagem em termos de ferramentas que o mesmo dispõe. Ao observar a Tabela 1 é possível notar que dentre as três categorias de funções, a mais presente foi a categoria de ferramentas de interatividade (In). Em 
geral, os autores citavam tais ferramentas de forma genérica ou listavam as principais (fórum, chat e wiki), porém, em onze delas o Wiki ganhou uma posição de importância fundamental para implementação do PBL em rede. Por isso, nestes casos o ícone genérico de interatividade foi substituído pelo de Wiki (W), explicitando a ferramenta central de interação do processo relatado na publicação. É o caso, por exemplo, de Sancho, Torrente e Fernandez (2012), que tiveram no Wiki a única ferramenta do Moodle utilizada no processo.

É importante notar que em algumas publicações, as funções de interatividade do Moodle não foram usadas ( $\mathrm{In} / \mathrm{W}$ ), mas isso não significa que não houve interatividade no processo, pois tais cursos eram híbridos, ou seja, ministrados parcialmente presencialmente.

A maioria das publicações, como pode ser visto na Tabela 1, declara explicitamente como sucesso a implementação de PBL em rede, indicados pelo ícone positivo (Rp). A omissão de tal declaração ou a apresentação de textos inconclusivos, seja porque o autor não foi assertivo na sua conclusão quanto ao sucesso da implementação de PBL em rede com o Moodle, implicou na não indicação de resultado positivo na Tabela 1 na linha da publicação correspondente (Rp). As publicações envolvendo descrição de software não tiveram sua conclusão indicada por não envolverem a implementação de PBL em rede, mas descreverem o desenvolvimento de ferramental para tal.

$\mathrm{O}$ que se destaca em termos de impressão dos autores sobre o PBL em rede foram os casos em que até foi indicado sucesso no processo (Rp), mas com ressalvas quanto ao aumento de trabalho e limitações do Moodle no atendimento às peculiaridades do processo PBL, casos de sucesso diferenciados com asterisco ( $\left.\mathrm{Rp}^{*}\right)$. Marti et al. (2015) destaca que o ferramental de avaliação do Moodle precisa ser aprimorado para atender as peculiaridades do processo PBL. Barnard-Ashton (2010) indica a falta de hábito dos alunos em atividades educacionais colaborativas através do Moodle, especialmente em cursos híbridos, como uma barreira importante a ser suplantada na implementação de PBL em rede. Podesta (2010) afirma que, apesar dos resultados positivos alcançados com PBL em rede, a sua implementação com o Moodle demanda bastante tempo e esforço adicional para tal. Zhen e Zhou (2011) acrescentam a estas ressalvas a necessidade de apoio institucional para viabilizar a logística voltada para a implementação de PBL em rede.

Os principais resultados da análise do grupo de publicações como um todo, realizada para identificação de eventuais vieses nos dados coletados, são mostrados na Figura 2. A Figura 2a apresenta o número de publicações selecionadas por cada ano, demonstrando que o interesse no uso do Moodle como ferramenta de suporte a PBL iniciou em meados nos anos 2000 e se manteve posteriormente. Tal dado indica boas perspectivas acadêmicas para a linha de pesquisa. Isso também 
é indicado por um grupo de pesquisa da universidade do Catar, que tem cinco publicações entre 2011 e 2016 (Ali, Samaka \& Shaban, 2011, Ali \& Samaka, 2013, Ali, Al-Dous \& Samaka, 2015, Al-Dous, Samaka, 2015, Ali, Wang, Samaka \& Miao, 2016), focados especialmente no desenvolvimento de ferramentais de suporte à PBL em rede.

A Figura $2 \mathrm{~b}$ indica em que áreas do conhecimento humano foram encontradas publicações, de acordo com a classificação da CAPES (2017), exceto as publicações relacionadas com apresentação de produto e experimentos envolvendo educação básica. Nota-se que as ciências biológicas concentram o maior número de publicações, mantendo a tradição de ser esta área a mais ativa no uso da metodologia PBL desde o seu surgimento, agora no contexto de PBL em rede. Mas também se nota um grande número de pesquisas aplicadas nas áreas de ciências exatas e engenharia. Considerando que as publicações de descrição de produto são oriundas da área de computação, o que as tornam uma publicação de ciências exatas, estas três áreas totalizam mais de $80 \%$ do total de publicações. O que é algo natural, pois são compostas pelas áreas afins com tecnologia (ciências exatas e engenharias) e a área que mais demanda a metodologia (ciências biológicas). A presença de publicações cujo foco é educação básica também demonstra a busca do uso de tecnologia para atender a necessidades peculiares da aplicação de PBL neste nível educacional (Huang \& Chuang, 2008; Boldini, et al., 2009; Shober \& Keller, 2012).

Surpreendentemente, foi detectada uma alta concentração de publicações oriundas da Espanha, país cujos pesquisadores foram os autores de mais de $40 \%$ das publicações selecionadas nesta pesquisa, como mostrado na Figura 2c. Essa busca de aprimoramento do ensino superior com uso de metodologias ativas de aprendizagem foi incentivada pela Declaração de Bolonha (European Commission, 2007), que iniciou um processo de convergência entre o sistema educacional espanhol com o da comunidade europeia (Garcia-Roubles, et al., 2009, p. 329). Tal destaque em publicações não está, portanto, ligado ao uso de tecnologia ou método específicos pelos autores espanhóis.

\section{CONSIDERAÇÕES FINAIS}

O estudo demonstrou que a investigação sobre implementação de metodologia PBL em rede é promissora, já conta com uma base de conhecimento razoavelmente sedimentada, porém tem muito a evoluir. Ao mesmo tempo em que autores como Garcia-Robles et al. (2009) e Diez-Riguera et al. (2010) deixam claro alcance de melhoria das notas e a diminuição de evasão em cursos baseados em PBL em rede, outros autores revelam as limitações e necessidades de aprimoramento que as tecnologias envolvidas apresentam para se otimizar o processo e atender 
as necessidades peculiares da metodologia (Marti et al, 2015; Zheng \& Zhou, 2011, Podesta, 2010; Barnard Ashton, 2010).

Apesar das considerações sobre o Moodle, a dificuldade que mais se fez mostrar na pesquisa foi a falta de padronização na interpretação do que é e o que constitui a metodologia Problem Based Learning (PBL), bem como sua diferenciação em relação a Project Based Learning (PjBL), especialmente dentre os autores das áreas de engenharias e ciências exatas, enquanto autores das áreas de ciências biológicas apresentam um melhor alinhamento com as principais publicações da área de PBL. Esta talvez seja uma barreira tão ou mais importante que a tecnológica, principalmente para educadores inexperientes no uso da metodologia, que podem facilmente se confundir em termos de definições e procedimentos na tentativa de aplicar a metodologia em sua prática pedagógica.

Podemos concluir, portanto, que embora seja uma alternativa pedagógica viável e interessante tecnológica e metodologicamente, há muito o que contribuir no sentido de tornar PBL em rede uma ferramenta para o processo de ensino-aprendizagem mais fácil e eficiente de se implantar. E isso pode ser feito resolvendo a falta de padronização quanto a definições, terminologia e protocolos relacionados com PBL, bem como com a adequação do Moodle para atender as peculiaridades da metodologia. Assim, a implementação de PBL em rede pode se dar de forma mais assertiva, especialmente por aqueles educadores que não tenham um maior embasamento teórico sobre a metodologia, e a sua aplicação pode ter maior produtividade, diminuindo o tempo e o esforço desprendido pelo professor para tal.

\section{REFERÊNCIAS}

Albanese, M. \& Mitchel, S. (1993). Problem-based learning: A review of literature on its outcomes and implementation issues. Academic Medicine, v. 68, p. 52-81.

Al-Dous, K. \& Samaka, M. (2015). The design and delivery of hybrid PBL sessions in Moodle. International Journal of Education and Information Technologies, v. 9, 2015, 105-114.

Ali, Z., Al-Dous, K. \& Samaka, K. (2015). Problem-based Learning Environments in Moodle: Implementation Approches. IEEE Global Engineering Education Conference.

Ali, Z. \& Samaka, M. (2013). ePBL: Design and implementation of a problem-based learning environment. IEEE Global Engineering Education Conference.

Ali, Z., Samaka, M. \& Shaban, K. (2011). A virtual problem based learning environment in Moodle. $5^{\text {th }}$ International Technology, Education and Development Conference, 2011.

Ali, Z., Wang, D. Samaka, M. \& Miao, Y. (2016). PLATE-PBL: Development and Implementation of a Script-based PBL Environment in Moodle. IEEE $16^{\text {th }}$ International Conference on Advanced Learning Technologies, 2016. 
Alonso, B., Arriba, L., Iniesta, M., Herrera, D., Santa Cruz, I. \& Sanz, M. (2011). Learning Environments and the construction of virtual dianostics and therapeutic guides in periodontology. $4^{\text {th }}$ International Conference of Education, Research and Innovation.

Audi, N. \& Escudero, D. (2012). Collaborative PBL \& Architectural Construction. IEEE Computers in Education.

Barnard-Ashton, P. (2010). Using wiki's in Problem Based Learning: e-Learner behavior and attitude. EDULEARN 2010 Conference.

Barrows, H. (1996). Problem-based learning in medicine and beyond: A brief overview. New Directions for Teaching and Learning, v. 68, p. 3-12.

Berkel, H.; Sherpbier, A., Hillen, H. \& Vleuten, C. V. D. (2010). Lessons from Problem-Based Learning, New York, USA: Oxford.

Bhardwaj, A., Naganla, K., Swe, K. M.M., Abas, A.B.L. (2015). Academic staff perspectives towards adoption of E-learning at Melaka Manipal Medical College: Has e-learning redefined our teaching model? Kathmandu University Medical Journal.

Biasutti, M. \& El-Deghaidy, H. (2015). Interdisciplinary project-based learning: an online wiki experience in teacher education. Technology, Pedagogy and Education.

Boldini, F., Braccini, M. R., Pouyioutas, P., Solomou, E \& Ioannou, C. (2009). The EUCLIDES Project - An On-Line Learning Portal Utilizing Problem-Based Learning. Advances in Web Based Learning - ICWL 2009, p. 73-77, 2009.

CAPES. (2017). Tabela de Áreas do Conhecimento, 2017. Disponível em: <http://www. capes.gov.br/avaliacao/instrumentos-de-apoio/tabela-de-areas-do-conhecimento-avaliacao>. Acesso em: 1 set. 2017.

Chan, C. W. (2006). COMPSoft - a plataform for online problem-based learning for health care professionals. [s.1.] Simon Fraser University.

Coquerel, P. R. S., Nunes, R. N., Oliveira, D. L. de Barros, C. S. de, Dantas Ferreira, A. C., Siqueira Garcia, L. C. et al. (2014). The influence of problem based learning in the teaching and learning process about relational psychomotricity in participants of an extension event of the study group in ludomotricity. $6^{\text {th }}$ International Conference on Education and New Learning Technologies.

Diez-Higuera, J. F., Antonio-Rodriguez, M., Martinez-Zarzuela, M., Gonzalez-Ortega, D. \& Boto-Giralda, D. et al. (2010). Adapting the telecommunication engineering curriculum to the EEES: a project based learning tied to several subjects. IEEE EDUCON Education Engineering.

European Commission. (2017). The Declaration of Bologna, 2007. Disponível em<http:// ec.europa.eu/education/policies/educ/bologna/bologna_en.html>. Acesso em: 1 de setembro de 2017.

Farias, G. F., Spanhol, F. J. \& Souza, M. V. (2016). The use of LMS to support PBL practices: a systematic review. Journal of Research \& Method in Education, v. 6, p. 3.

Farias, G. F., Spanhol, F. J. \& Souza, M. V. (2017). Uma revisão narrativa sobre soluções de aprendizagem em rede baseada em problemas, in Educação Fora da Caixa - Tendência para Educação do Século XXI, Florianópolis: Via UFSC. 
Fernandez-Borras, J. Torrella, J. R., Viscor, G. \& Pagés, T. (2015). Evaluation of a lesson resource for problem-base/ lended-learning in the undergraduate course of applied animal biology. $8^{\text {th }}$ International Conference of Education, Research and Innovation.

Garcia-Robles, R., Diaz-Del Rio, F., Vicente-Diaz, S. \& Linhares-Barranco, A. (2009). An eLearning Standard Approach for Supporting PBL in Computer Engineering. IEEE Transactions on Education, v. 52, n. 3.

Giacomozzi, C., Cordella, D., Rogante, R., Zampolini, M. et al. (2006). A European E-Learning Experience in Upper Limb Telereha-bilitation. TELEHEALT.

Giacomozzi C., Campobello, G., Huijgens, B. Ilsbroukx, S., Macellari, V., Magni, R., etal. (2008). Tele-rehabilitation and e-learning: the HELLODOC educational experien$c e$. Ann Ist Super Sanità 2008, v. 44, n. 2:145-153.

Gomez-Lahoz, C., Garcia-Rubio, A., Paz Gacia, J. M., Garcia-Herruzo, F. \& Vereda-Alonso, C. 2010). The challenge of tutoring autonomous and collaborative learning in virtual universities. INTED2010 Conference.

Gonzalez-Sancho, J. M., Sanchez-Pacheco, A., Lasa, M., Molina, S., Vara, F. \&, Del Peso, L. (2013). The use of an active learning approach to teach metabolism to students of nutrition and dietetics. Biochemistry and Molecular Biology Education.

Gutierrez, G. \& Berciano, A. (2012). A teaching experiment about the influence of using PBL on the mathematical competence on prospective teachers of early childhood education. International Conference on New Horizons in Education.

Halbaut, L., Pozo Carrascosa, A. Del., Roig Montblanch, Viscasillas Clerch, A., Juv, J. (Josep), Arztegui Trenchs, M. et al. (2010). La formulación magistral: adquisición de competencias mediante ABP y estrategias no presenciales. Ars Pharmaceutica 2010, v. 51, n. 2, p. 75-8.

Huang, C. J.; Chuang, Y. T. (2008). Supporting the development of collaborative problem-based learning environments with an intelligent diagnosis tool. Science Direct, 35, p. 622-631.

Leon, L. B. De; Onófrio, F. de Q. (2015). Aprendizagem Baseada em Problemas na Graduação Médica - Uma Revisão da Literatura Atual. Revista Brasileira de Educação Médica, v. 39, n. 4, p. 614-619.

Martí, E., Gil, D., Gurguí, A., Hernández-Sabaté A., Rocarías, J. \& Poveda F. (2015). $P B L$ on line: A proposal for the organization, part-time monitoring and assessment of PBL group activities. Journal of Technology and Science Education, v. 5.

Martinez, F. Herrero, L.C., \& Pablo, S.D. (2011). Project-Based Learning and Rubrics in the Teaching of Power Supplies and Photovoltaic Electricity, IEEE Transactions on Education, v. 54, n. 1.

Mezzari, A. (2011). O uso da aprendizagem baseada em problemas (ABP) como reforço ao ensino presencial utilizando o ambiente de aprendizagem Moodle. Revista brasileira de educação médica, v. 35, n. 1: 114-121.

Miao, Y., Holst, S., Holmer, T., Fleschutz, J. M. \& Zentel, P. (2000). An activity-oriented approach to visually structured knowledge representation for problem-based learning in 
virtual learning environments. Anais. International Conference on Information in the 21 Century: Emerging Technologies and New Challenges.

Moreno-Brea, M. J., Arellano-Lopez, J. M. \& Arufe-Martinez, M. I. (2011). Exploring different types of collaborative assignments in toxicology using wikis. $4^{\text {th }}$ International Conference of Education, Research and Innovation.

Onan, A., Turan, S. \& Gurlen, E. (2014). Do moodle reports and logs meet the needs of educational supervision? $9^{\text {th }}$ Iberian Conference on Information Systems, 2014.

Osinaga, X, Mujika, M. \& Manso, A. (2011a). Computer-aided design: problem-based learning approach, $4^{\text {th }}$ International Conference of Education, Research and Innovation. Osinaga, X, Mujika, M. \& Manso, A. (2011b). Problem-based learning, implementation and qualitative study in computer aided design subject. $5^{\text {th }}$ International Technology, Education and Development Conference.

Podesta, L. (2010). Course Management Systems: do they improve the quality of teaching and learning? Joint International IGIP-SEFI Annual Conference, 2010.

Reguero-Gomez, A., Busoch-Morlin, C. B., Busoch, C. \& Sanchez-Bao, R. (2011). Experiencias en la enseñanza semipresencial en el plan de estudio de ingeniería biomédica en el "Instituto Superior Politécnico José Antonio Echeverría". V Latin Aerican Congress On Biomedical Engineering.

Reis, L. O., Ikari, O., Taha-Neto, K. A., Gugliotta, A \& Denardi, F. (2014). Delivery of a urology online course using moodleversus didactic lectures methodsLeonardo. International Journal of Medical Informatics.

Sancho, P., Torrente, J., Marchiori, E. J., \& Fernandez-Manjon, B. (2011). Enhancing moodle to support problem based learning. The Nucleo experience. IEEE Global Engineering Education Conference.

Sancho, P., Torrente, J. \& Fernandez-Manjon, B. (2012) MareMonstrum: a contribution to empirical research about how the use of MUVEs may improve student's motivation. Journal of Universal Computer Science, v. 18, n. 18.

Saydam, S., Mitra, R., Daly, C. \& Hagan, P. (2009). A collaborative approach to mining education in Australia. The International Journal of Learning, v. 16, n. 3.

Sein-Echaluce, M. L., Aguado, P. M., Esteban-Escao, J. esteban-Sanchez, A., Florentin, P., Gracia-Gomez, M. C., et al. (2015). Design of adaptive experiences in higher education through a learning management system. $3^{\text {rd }}$ International Conference on Technological Ecosystems for Enhancing Multiculturality.

Sevil, J., Terrer, J. \& Llanas, S. (2012). Application of small mobile robots as improvement of the motivation of engineering students. $4^{\text {th }}$ International Conference on Education and New Learning Technologies.

Shober, A. \& Keller, L. (2012). Impact factors for learner motivation in Blended Learning environments. International Journal of Emerging Technologies in Learning, v. 7.

Stebbins, S., Bagheri, N., Perrie, K., Blyth, P., \& McDonald, J. (2012). Blended learning and curriculum renewal across three medical schools: The rheumatology module at the 
University of Otago. Australasian Journal of Educational Technology, v. 28, n. 7, p. 1176-1189.

Suebnukarn, S. \& Haddawy, P. (2007). COMET: A collaborative tutoring system for medical problem-based learning. IEEE Intelligent Systems, v. 22, n. 4, p. 70-77.

Tiantong, M. \& Teemuangsai, S.(2013). The four scaffolding modules for collaborative problem-based learning through the computer network on Moodle LMS for the computer programming course. International Education Studies, v. 6, n. 5.

Tirado, F. \& Santos, G. (2014). Examining the Relationship Between Epistemic Activity and Academic Achievement. $9^{\text {th }}$ International Conference on Computer Science \& Education.

Wood, D. F. (2003). ABC of learning and teaching in medicine: Problem based learning. BMJ, v. 326, n. 2, p. 328-330.

Woods, D. R. (1994). Problem-Based Learning - How to Gain the Most from PBL. Hamilton, ON, Canadá: Grifin.

Woods, D. R. (2006). Preparing for PBL. Waterdown, ON, Canadá: Don Woods, 2006. Zheng, A. \& Zhou, Y. (2011). An inductive interactive and adaptive hybrid problem-based learning methodology: Application to statistics. Journal of Engineering Science and Technology, v. 6, n. 5, p. 639. 
